\title{
Multi-Level Trust Privacy Preserving in Data Mining
}

\author{
A. Damodar, Srinivasulu Reddy, K. David Raju
}

\begin{abstract}
User interfaces for computer code applications will are available in a range of formats, starting from command-line, graphical, net application, and even voice. whereas the foremost well-liked user interfaces embody graphical and web-based applications, often the requirement arises for an alternate interface. whether or not thanks to multi-threaded complexness, synchronic property, or details encompassing execution of the service, a conversation larva primarily based interface might suit the requirement. Chat bots usually give a text-based computer program, permitting the user to kind commands and receive text furthermore as text to speech response. Chat bots square measure sometimes a stateful services, basic cognitive process previous commands (and maybe even conversation) so as to produce practicality. once chat larva technology is integrated with well-liked net services it may be utilised firmly by a fair larger audience.
\end{abstract}

Keywords : multithreaded,interface,chat bot,

\section{INTRODUCTION}

There are numerous applications related to information mining which manage protection concern. Bank exchanges, healing facility records, and web movement are a few cases. Information mining in such security touchy stage is confronting developing issues. We require creating procedures that are touchy to the security issue. This has expanded the improvement of a class of calculations. This paper comprises several methods for PPDM by irritating the information while safeguarding the hidden powerful properties. Clamor is added to safeguard the information while making beyond any doubt that this clamor protects the flag from the information so that the examples can at present be effectively assessed. Web gathers the information on an expansive scale. Nonetheless, taking such benefits are worries about data security.

Due to this, a few people may choose to give wrong data in danger of security issues, or they may basically decline to disinter any information whatsoever. Apparatuses like Information-investigation and learning investigation utilizing these data may subsequently figure comes about with absence of rightness. After data are gathered, they can be utilized as a part of numerous Information-examination and learn investigation calculations, the result of which can profit the data authorities as well as their clients. A, for the most part, utilized valuable information investigation

Revised Manuscript Received on 14 August, 2019.

A.Damodar, Asst. Professor, Dept. of CSE., Malla Reddy Engineering College for Women, Hyderabad, India

Srinivasulu Reddy, Asst. Professor, Dept. of CSE., Malla Reddy Engineering College for Women, Hyderabad, India

K. David Raju, Assoc. Professor, Dept. of CSE., St.Peter's Engineering College, Hyderabad, India. technique is Data Mining. Information mining alludes to separating learning from the broad measure of data; its point is to investigate information, patterns, and designs, and so on from a broad set of data. In this paper, our principle plan is on chosen information mining assessment. At the point when the data in the database are a shroud, we need to search out how to settle on choice trees $b$.

The inner (most trusted) information mining is may be finished by government or business areas, yet at some point, they need to discharge their information to general society and furthermore irritate it more. The mining division approach both less bothered inside duplicates and more bothered open duplicates. Regardless of the possibility that they have the two examples of interior duplicates and bothered open duplicates, they can't reproduce the first information. General society will have every one of the privileges of the information mining division if the private duplicate of information is spilled socially. In any case, it is hard to open to reproduce unique information precisely by utilizing just spilled inward duplicates. New difficulties are made for dissemination based PPDM because of the new part of MLT - Multilevel Trust. As a contrast with singular level trust, numerous circulated set of the same information is accessible at a various different level to aggressors. less irritated duplicates can be gotten to by more confided in information mineworker. This information digger may likewise approach bring down trust levels to annoyed duplicates. There are many intends to get to various irritated duplicates. Past the utmost set by information proprietor, it is conceivable to information excavator to remake unique information.

\section{BACKGROUND INFORMATION}

This assault is called assorted variety assault. Assorted variety assault incorporates the situation conniving assault in which foes join their duplicates with the end goal of assaulting. At some point the situation in which aggressor brings open data to perform an assault on itself. It is a noteworthy test in MLT-PPDM issue to avert assorted variety assault. To take care of the issue, effectively numerous arrangements have been proposed by the analysts who additionally get ordered into two classifications in light of the protection which they will give. The primary class is multi-party calculation security approach which gives an awesome level of security to the protection by just giving them the data which they have mined as opposed to some other inclinations to information. These calculations are extremely successful however they are exceptionally costly and seldom utilized. to maintain a strategic distance from the 


\section{Multi-Level Trust Privacy Preserving in Data Mining}

high-cost different arrangements have planned like developing choice trees over level information while kimplies bunching and affiliation administer digging are intended for the vertical information mining.

The associations can utilize the other way to deal with keeping their private information from the pernicious clients when the information is accommodated the examination reason. The irritation will guarantee the respectability of the information. For the most part in the field of the restorative part, the irritation can be utilized successfully in such segments for the avoidance from the spilling of the information duplicates. there are different strategies for the annoyance accessible which give the distinctive level of securities.

It likewise has some negative effects which can be dealt with and irritated duplicates can be utilized effectively. In today's situation, for the most part, all the work is completed on the web, what's more, the security to this information has turned into the most critical perspective as the data accessible online can be secret what's more, can be abused by somebody. So to keep away from this circumstance, different strategies are getting actualized and by consolidating this every single new system the information is kept confidential. the principle challenge is the way to ensure this information amid the mining procedure and furthermore acquire the exact outcomes as when required. Mainly encryption and unscrambling are the real angles that need to be considered while applying the different strategies.

Protection saving strategies can be grouped in view of following attributes:

1. Data mining Scenario

2. Data mining tasks

3. Data Contribution

4. Data types

5. Privacy definition

6. Protection method

\section{CONTRIBUTIONS}

We will make the accompanying commitments: We will grow the extent of PPDM to multilevel trust, by beating the single-level trust which is as of now existing. MLT-PPDM will give an extraordinary adaptability which will permit information proprietors to make their own particular irritated duplicates with various levels of security. Our principle plan will be to give the multi-level trust to the irritated duplicates. In MLT-PPDM, information excavators will have the arrangement to get to numerous annoyed duplicates. On the off chance that numerous annoyed duplicates get joined together, decent variety assaults can be performed by information mineworkers to re-arrange the unique information more impeccably than what is permitted by the information proprietor. Defeating such difficulties is the key-point we will give a solid answer for it. We will give a suitable answer for this test by appropriately sorting out the irritation crosswise over duplicates at various put stock in levels. We will give an answer which will get maintained against assorted variety assaults. We give the calculation which will effectively deal with the genuine information of the private clients. Our answer will give the office of creating the annoyed duplicates effortlessly to the information proprietors. This property will give most extreme adaptability to information proprietors



A. Proposed System Architecture:

\section{Figure 1: Design of MLT_PPDM}

Here we depict our framework engineering. The principal modules in our engineering are User, security System, Fraud Client, Admin, and Database. The client gets a login to the framework through a secure login. The client is given a remarkable id to login. Security framework incorporates calculation which is utilized for security reason. Because of security extortion, the client can't get to the information. Along these lines get information is fizzled. The administrator is the primary module which keeps up the entire framework. Which move is to be made is chosen by the administrator. Database resembles a holder which contains all the information. Information of client is put away in the database. The memory of database is substantial. In this manner, the information in the database is secured.

All the procedure is bi-directional.

\section{B. Flow Diagram}

Where \# Q \# 0 is the state where the main data set is stored on which the processing needs to be done to provide the privacy on the data on various levels as per the requirements.

Where,

$$
\text { Q 1 = Data Set with Noise[D(ni)] }
$$

Where \# Q \# 1 is the state where the noise in the form of different techniques is added to the main dataset for the different privacy levels.

Where,

$$
\text { Q } 2 \text { = Perturbed Copies of data [DN p(ni)] }
$$

Where \# Q \# 2 is the state where the data set with noise get distributed into different perturbed copies where each individual perturbed copy

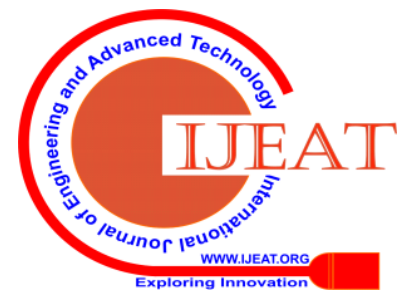


contains a part of data added with noise in the form of an encrypted copy or the perturbed copy which is further sent to next state for the further processing.

Where,

Q 3 = Apply key generation on perturbed copies[KG].

$$
\begin{gathered}
\mathrm{Q} 4=\{\mathrm{P} \mathrm{U}+\mathrm{P} \mathrm{R}\} \\
\mathrm{Q} 5=\{\mathrm{OD}\}
\end{gathered}
$$

Where ' $S$ ' is the main set of states through which the system is going to pass through its life-cycle and the data is going to

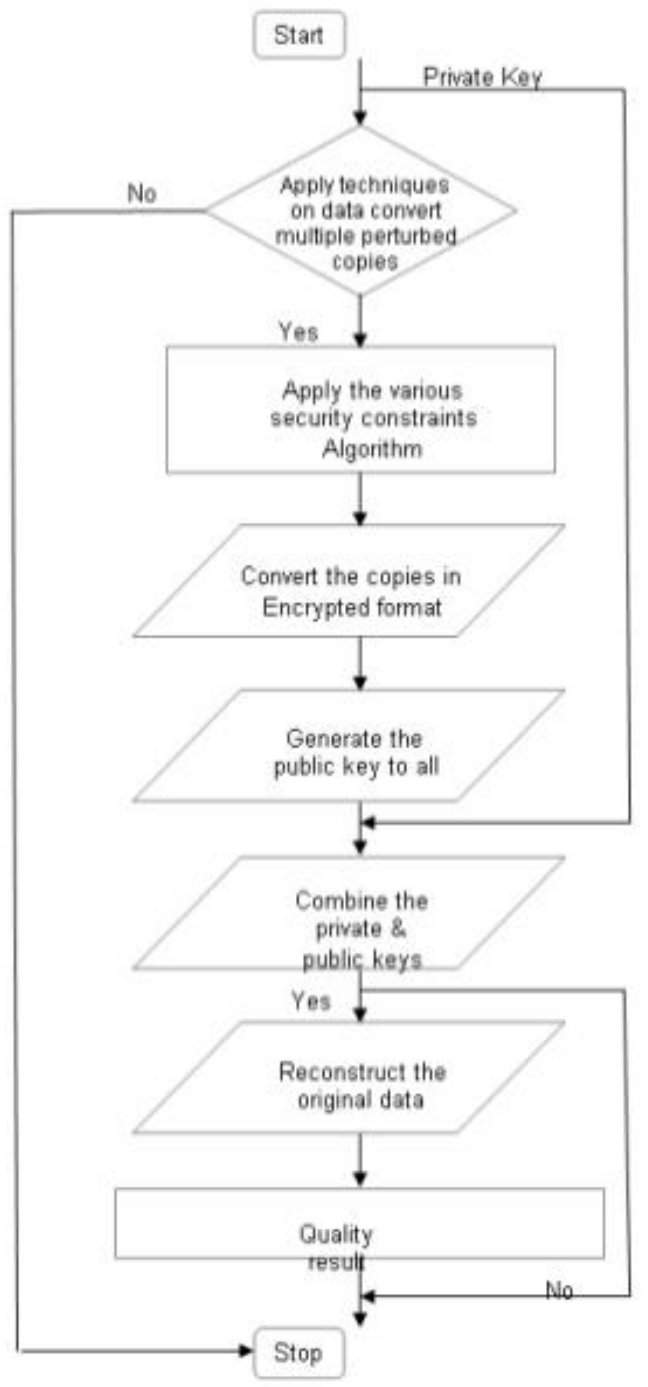

pass through the respected states.

Where,

$$
\text { Q } 0=\text { Data } \operatorname{Set}[\mathrm{D}(\mathrm{n})]
$$

\section{Mathematical Model}

Where 'Q3' is the state where the key generation technique is used to further encrypt the multi-level privacy on the different perturbed copies of the main confidential data and a public key is applied to all the copies for the further processing.

Where,

Q 4 = Matching the combination of keys [P U + P R]

So, the above mechanisms can be further elaborated as:

Let,

$\mathrm{S}=\{\mathrm{Q} 0, \mathrm{Q} 1, \mathrm{Q} 2, \mathrm{Q} 3, \mathrm{Q} 4, \mathrm{Q} 5\}$

$\mathrm{Q} 0=\{\mathrm{D}(\mathrm{n})\}$
$\mathrm{Q} 1=\{\mathrm{D}(\mathrm{ni})\}$

$\mathrm{Q} 2=\{\mathrm{DN}$ p(ni) $\}$

Q $3=\{K G\}$

Where \# Q \# 4 is the state where the combination of public key and the private key is matched. If the key gets matched then the original data can be reconstructed otherwise the process will get terminated in the same instance.

Where,

$$
\text { Q } 5 \text { = Original Data[OD] }
$$

Where \# Q \# 5 is the state where if the keys are matched in the previous state then the original data will get generated in this state to the genuine user otherwise the process will not execute further.

So by the abbreviations above, we can get

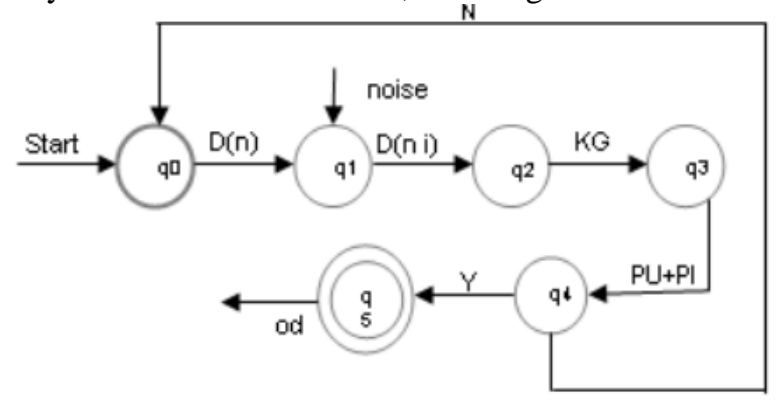

Encrypt $=\{\mathrm{KG}[\mathrm{DN} \mathrm{p}(\mathrm{ni})]\}$

So, the above-abbreviated algorithm can be used on the whole data set to provide the multi-level privacy of the Genuine User's confidential data set.

\section{CONCLUSION}

Security protecting information mining can possibly increment the compass and advantages of information mining innovation. Be that as it may, we should have the capacity to legitimize that protection is safeguarded. For this, we should have the capacity to convey what we mean by security safeguarding. The present blend of definition, with each paper having its own meaning of what protection is kept up, will prompt perplexity among potential adopters of the innovation. The paper displays some proposal for characterizing and checking protection conservation. We demonstrate how this identity with both protections approach and practice in the more extensive group, and to strategies in protection saving information mining.

This is in no way, shape or forms the conclusive word regarding the matter. While a few measures, for example, the differential entropy metric of, have clear numerical establishment and Application, others have solid potential for facilitating improvement. Adopting a basic system for the discourse of protection safeguarding will empower cutting-edge information mining innovation to make considerable advances in reducing security concerns. In this paper the Expanded PPDM to multilevel confide in (MLT) is presented and we expand the extent of PPDM, wherein existing framework single level trust is accessible. Multilevel Trust Security Preserving Data Mining permits to create multilevel confide in divided duplicates of information for created by information proprietor.

Published By:

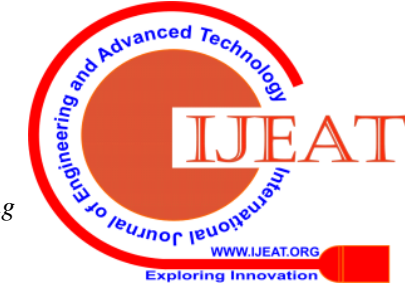




\section{Multi-Level Trust Privacy Preserving in Data Mining}

The primary test is to counteract information excavators or assailant from consolidating the divided duplicates of different put stock level to frame unique informational collection created by information proprietor. We discover answer for this test by including the clamor in the numerous divided duplicates at various put stock level. Our best piece of this examination is we enable information proprietor to create divided duplicates of its informational collection at arbitrary trust level on the base of demand. This arrangement gives the greatest adaptability to the information proprietor.

\section{REFERENCES}

1. S. M. Metev and V. P. Veiko, Laser Assisted Microtechnology, 2nd ed., R. M. Osgood, Jr., Ed. Berlin, Germany: Springer-Verlag, 1998.

2. J. Breckling, Ed., The Analysis of Directional Time Series: Applications to Wind Speed and Direction, ser. Lecture Notes in Statistics. Berlin, Germany: Springer, 1989, vol. 61.

3. S. Zhang, C. Zhu, J. K. O. Sin, and P. K. T. Mok, A novel ultrathin elevated channel low-temperature poly-Si TFT, IEEE Electron Device Lett., vol. 20, pp. 569571, Nov. 1999.

4. M. Wegmuller, J. P. von der Weid, P. Oberson, and N. Gisin, High-resolution fiber distributed measurements with coherent OFDR, in Proc. ECOC00, 2000, paper 11.3.4, p. 109.

5. R. E. Sorace, V. S. Reinhardt, and S. A. Vaughn, High-speed digital-to-RF converter, U.S. Patent 5668 842, Sept. 16, 1997. (2002) The IEEE website. [Online]. Available: http://www.ieee.org/

6. M.Shell.(2002) IEEEtran homepage on CTAN. [Online].Available: http://www.ctan.org/tex-

7. archive/macros/latex/contrib/supported/IEEEtran/

8. FLEXChip Signal Processor (MC68175/D), Motorola, 1996.

9. "PDCA12-70 data sheet," Opto Speed SA, Mezzovico, Switzerland.

10. A. Karnik, Performance of TCP congestion control with rate feedback: TCP/ABR and rate adaptive TCP/IP, M. Eng. thesis, Indian Institute of Science, Bangalore, India, Jan. 1999.

11. J. Padhye, V. Firoiu, and D. Towsley, A stochastic model of TCP Reno congestion avoidance and control, Univ. of Massachusetts, Amherst, MA CMPSCI Tech. Rep. 99-02, 1999.

12. Wireless LAN Medium Access Control (MAC) and Physical Layer (PHY) Specification, IEEE Std. 802.11, 1997.

13. L. Sweeney. Computational Disclosure Control: A Primer on Data Privacy Protection. Ph.D. thesis, Massachusetts Institute of Technology, 2001.

14. A. C. Yao. How to generate and exchange secrets. In Proceedings of the 27th IEEE Symposium on Foundations of Computer Science, pages 162167. IEEE, 1986

15. D. Agrawal and C.C. Aggarwal, On the Design and Quanti cation of Privacy Preserving Data Mining Algorithms, Proc. 20th ACM SIGMODSIGACT-SIGART Symp. Principles of Database Systems (PODS 01), pp. 247-255, May 2001.

16. . K. Chen and L. Liu, Privacy-Preserving Data Classi cation with Rotation Perturbation, Proc. IEEE Fifth Intl Conf. Data Mining, 2005 K. Liu, H. Kargupta, and J. Ryan, Random Projection-Based Multiplicative Data Perturbation for Privacy Preserving Distributed Data Mining, IEEE Trans. Knowledge and Data Eng., vol. 18, no. 1, pp. 92-106, Jan. 2006.

17. P. Samarati and L. Sweeney. Protecting privacy when disclosing information: k-anonymity and its enforcement through generalization and suppression. In Proceedings of the IEEE Symposium on Research in Security and Privacy, May 1998.

18. N. Nithiyanandam, K. Venkatesh, M. Rajesh, Transfer The Levels Of The Monitored Carbon, Nitrogen Gases From The Industries, International Journal of Recent Technology and Engineering, Volume-7 Issue-6S3 April, 2019.

19. Sivanesh Kumar, A., Brittoraj, S., Rajesh, M., Implementation of RFID with internet of things, Journal of Recent Technology and Engineering, Volume-7 Issue-6S3 April, 2019.

20. Rajesh, M., Sairam, R., Big data and health care system using mlearningJournal of Recent Technology and Engineering, Volume-7 Issue-6S3 April, 2019.

21. Rajesh, M., and J. M. Gnanasekar. "Path Observation Based Physical Routing Protocol for Wireless Ad Hoc Networks." Wireless Personal Communications 97.1 (2017): 1267-1289. 